SESSION VIII. INVENTORY AND DISTRIBUTION - Chair: JiPi De Greve 


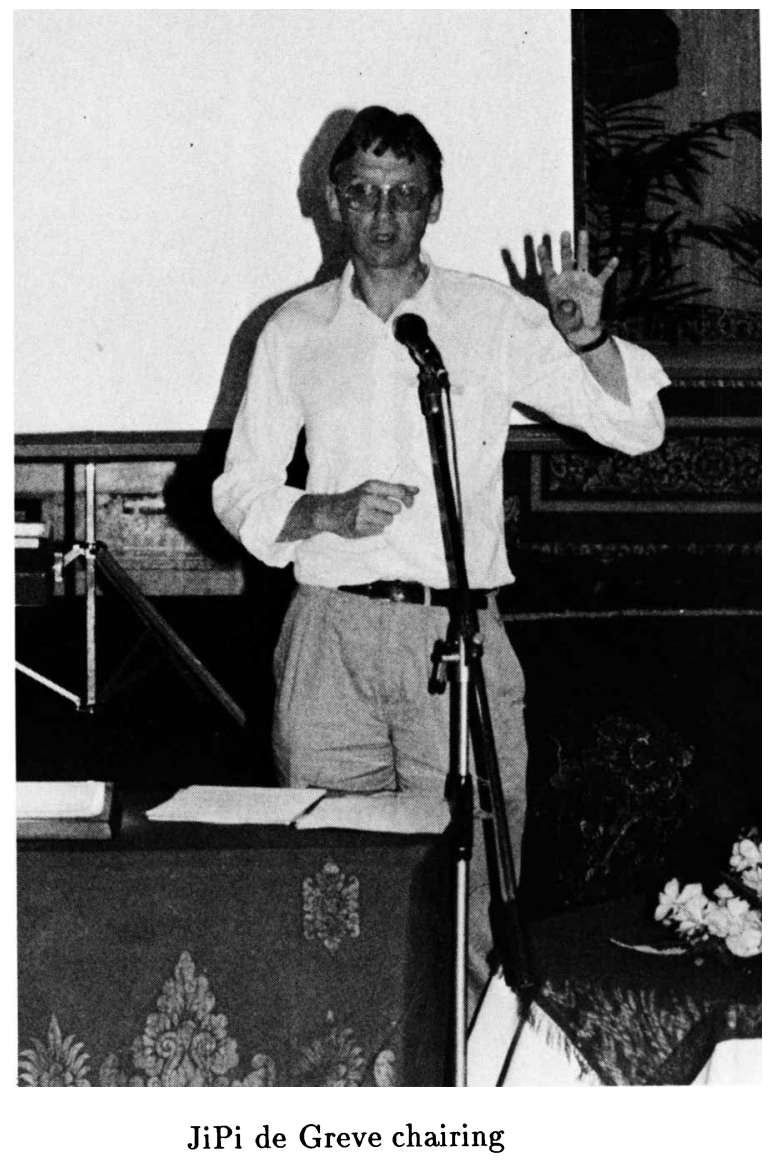




\title{
WOLF-RAYET STARS IN NEARBY GALAXIES: FACTS AND FANCIES
}

\author{
Philip Massey and Taft E. Armandroff \\ Kitt Peak National Observatory \\ National Optical Astronomy Observatories
}

\begin{abstract}
Surveys for Wolf-Rayet stars in nearby galaxies are briefly reviewed. The completeness and yield of these surveys are discussed in light of recent follow-up spectroscopy. A critical evaluation is made of our current knowledge of the Wolf-Rayet population in nearby galaxies, particularly the $\mathrm{WC} / \mathrm{WN}$ ratio, the WR/O ratio, the WR surface density, and how these quantities vary within a galaxy and between galaxies, particularly as a function of metallicity. We compare the spectroscopic properties of Galactic and Magellanic Cloud WR stars with those in the more distant systems.
\end{abstract}

\section{INTRODUCTION}

The last decade saw a great flurry of investigations of Wolf-Rayet stars in nearby galaxies of the Local Group. Such studies have provided a great deal of new information on the numbers of WR stars found in these galaxies, their types (WC/WN), their distributions, and their spectroscopic properties. A partial list of these studies includes Massey and Conti (1983), Moffat and Shara (1983), Armandroff and Massey (1985), Massey, Armandroff, and Conti (1986), Massey, Conti, Moffat, and Shara (1987), Massey, Conti, and Armandroff (1987), Moffat and Shara (1987), and Azzopardi, Lequeux, and Maeder (1988); reviews may be found in Massey $(1985,1986)$ and Conti $(1988)$.

In preparing today's review talk it became clear to us that not only have these studies resulted in a certain body of "facts", but have also been responsible for a certain number of "fancies". Despite claims of incompleteness and caveats that are mentioned in many of these papers, interpretations of dubious authenticity have become part of our common knowledge. For instance, Massey and Conti (1983) found evidence of a strong galactocentric gradient in the relative number of WC/WN stars in M33, with most WC's found towards the center of the galaxy and most WN's located in the outer regions; this is commonly attributed to a metallicity gradient, although a variety of other explanations (such as a gradient in the IMF) may be responsible. Before we get into arguments as to the cause of this gradient, we might want to consider whether such a gradient exists at all given newer data. Then we might want to examine how well established the metallicity gradient is in M33. Finally we might do well to ask whether the WC/WN ratio at a point in M33 where the metallicity matches that of the LMC is the same as the WC/WN ratio in the LMC. The members of this audience who are regular attendees of these beach symposia may remember Conti's (1982) "An observer's view of stellar theory" diagram from the Cozumel WR meeting, in which a tiny fraction of the rain of observational data falls into the theoretician's black box (held up by the edifice of assumptions and pillar of avoidance) resulting in a steady outflow of predictions leading to muddied waters. It is our self-appointed misson today to critically examine one

K. A. van der Hucht and B. Hidayat (eds.),

Wolf-Rayet Stars and Interrelations with Other Massive Stars in Galaxies, 575-586.

(c) 1991 IAU. Printed in the Netherlands. 
particular aspect of this process, namely the observational basis for the new mythology. In doing so, it is worth remembering that we are groping in the dark in these studiesno theoretician sat down in 1975 , predicted that the WC/WN ratio (say) would be a monotonic function of metallicity, and encouraged observers to confirm or refute this. Instead, all such predictions have been post facto, and the observations we have made over the years have been at best guided by what a recent Kitt Peak summer student described as "educated, intuitional whims." Today we will examine to what extent these factos are in fact facts. Much of the work described was done in collaboration with Peter Conti, who nevertheless should be considered immune from prosecution for any of the material discussed today.

The motivation for studying Wolf-Rayet stars in nearby galaxies is two-fold: (a) what we can learn about the parent galaxies themselves (in which we are basically using WR stars as probes of the massive star content), and (b) what we can learn about massive star evolution (for instance, what evolutionary paths lead to the WC and WN types and how is this affected by such factors as metallicity). Such studies have undergone a revolution in the few years since the Porto Heli meeting on luminous stars in galaxies due to the implementation of multi-object fiber feeds on the world's largest telescopes, coupled to the routine availability of low read-noise, high quantum efficiency CCD's for spectroscopy. Thus in a single night of observations on the Mayall 4-m, it is possible to obtain spectra of $>100$ faint objects in a nearby galaxy (cf. Barden and Massey 1988). At the time of the Porto Heli meeting, the recent advent of CCD's on large telescopes had allowed sensitive surveys for WR stars to be carried out in NGC6822, IC1613, M33, and M31; the new instrumentation that has come along since that time has allowed the spectroscopy to finally catch up with the imaging surveys. The major punchlines of this spectroscopic work can be characterized as follows: (1) many of the lower significance WR candidates found from the CCD surveys are now spectroscopically confirmed, demonstrating that the older photographic surveys were woefully incomplete, (2) the WC/WN ratio in M31 appears to be no higher than that in M33, suggesting that whatever controls this ratio is not simply metallicity, and (3) the spectra of WR stars in NGC6822, IC1613, and M33 are remarkably similar to those in the Milky Way and Magellanic Clouds; the jury is still out on the M31 WR stars.

Below we list the major facts and fancies that have surfaced during the past decade of research; in some instances, there are "strong" and "weak" versions of the same law.

- The WC/WN ratio is a monotonic function of metallicity. - The WC/WNE ratio is a monotonic function of metallicity. - The WR/O ratio is a monotonic function of metallicity. - The $(\mathrm{WC}+\mathrm{WNE}) / \mathrm{O}$ ratio is a monotonic function of metallicity.

- WCL stars are exceedingly rare and are found only in the metal-rich central regions of M31 and the Milky Way.

- M33 WR stars are not quite like any others in the universe.

- FWHM of lines for a given WC subtype is a function of metallicity in M33.

- M31 WR stars are not quite like any others in the universe.

- The numbers and types of WR stars in all galaxies of the Local Group are accurately known, or well-established correction factors can be applied.

- The number of WR stars per unit anything ( $\mathrm{kpc}^{2}$, mass) is a factor of 10 lower in M31 than it is in the Milky Way, conclusively demonstrating that there are few massive stars present in M31. 
We will discuss the observational reality of the facts and fancies given above in two general categories. (1) What do we know about the numbers of WR stars in Local Group galaxies (including the WC/WN ratio, the number per unit area or mass)? (2) What do we know about the spectra of WR stars in these galaxies? Are late-type WC stars present and, if so, are they really restricted to the central regions of metal-rich systems (e.g., M31)? More generally let us ask if the line widths and strengths are the same among these extra-galactic systems. This is the same as asking if the stellar wind laws are the same.

\section{NUMBERS AND DISTRIBUTION OF WR STARS}

Galactic WR stars have been found by a variety of means, mostly incidentally as part of general spectroscopic surveys (e.g., the Henry Draper Catalogue). Roughly 160 Galactic WR stars are known (van der Hucht et al. 1981). Doubtless there are lightly reddened WR stars of 12th mag and brighter still to be found, and elsewhere in these proceedings Shara describes a dedicated search for WR stars in the Galaxy using optical techniques. Our knowledge of the WR content of the Galaxy is believed to be complete within 3 $\mathrm{kpc}$ of the sun or so. In a recent paper, Conti and Vacca (1990) derive distances to Galactic WR stars using spectroscopic parallax and improved estimates of the reddening corrections. Within $2.5 \mathrm{kpc}$ of the sun there are $48 \mathrm{WR}$ stars known, 24 of WN type and 24 of WC type.

The Magellanic Cloud WR stars have been found primarily using objective prism plates with a broad $\lambda 4650 / 4686$ interference filter used to alleviate crowding; details can be found in Breysacher (1981) and Azzopardi and Breysacher (1979). Roughly 100 are known in the LMC and 8 in the SMC. While occasionally a new one shows up in the LMC, these numbers are probably essentially complete. Of the 100 -odd WR stars in the LMC, $\approx 20 \%$ are of WC type. Of the $8 \mathrm{WR}$ stars in the SMC, only 1 is of WC type.

It is worthwhile to pause for a moment here and reflect on what the numbers from these three galaxies alone tell us. First of all, we see that the total number of WR stars in each of these galaxies does not scale in a simple way with mass or area surveyed. The number of WR stars per $\mathrm{kpc}^{2}$ is 2.3 in the solar neighborhood, and something like 2 in the LMC, but only 1 in the SMC. Of course, we don't know if we have considered equal volumes or not, given the uncertainty in the depth extensions of the Clouds. Per unit mass it would appear that the number of WR stars is down about a factor of 3 in the SMC compared to the LMC (Azzopardi and Breysacher 1979), although clearly the masses of these galaxies are rather uncertain. These numbers may be telling us that the number of massive star progenitors is down by a factor of 2 or 3 per unit something in the SMC compared to that of the LMC, implying that the star formation rate is lower in the SMC...or they may not.

What about the ratio of WC to WN stars? The progression in this ratio from the Galaxy to the LMC to the SMC is in the same sense as the change in metallicity. The ratio of WC to WN stars is 1.0 in the solar neighborhood, 0.2 in the LMC, and 0.1 in the SMC; the "metallicity" of the Milky Way is the highest, with that of the LMC down by a factor of 0.4 dex and that of the SMC down by another factor of $0.4 \mathrm{dex}$, using the oxygen abundance as the metallicity indicator. But hold it, there is something remarkably fishy here. In the SMC we are dealing with (very) small number statistics-there is exactly 1 WC star among the $8 \mathrm{WR}$ stars. Given the stochastic probability of finding exactly one of anything in something the size of a galaxy, we must at least take the uncertainty in this 
quantity to be $\sqrt{N}$ : if we looked at some slightly different time, would we see zero or two WC stars? In the case of there being two WC stars, the ratio in the SMC changes to 0.3, larger than that of the LMC. Thus the "progression" from the Milky Way to the LMC to the SMC is in fact meaningless! What is fair to say is that the relative number of WC to WN stars is far lower in the Magellanic Clouds than it is in the solar neighborhood, but to say any more than that ignores the inherent uncertainties in the small numbers for the SMC. We emphasize that we are not saying that the current numbers of WC and WN stars are poorly known in the SMC-just that there is a certain amount of luck in living at this particular time if we are seeing exactly one star whose lifetime is so short compared to a Hubble time. While we are debunking the "progression" myth of the Milky Way $\rightarrow \mathrm{LMC} \rightarrow \mathrm{SMC}$, let us note that whatever it is that is primarily responsible for driving the stellar wind, it is unlikely to be oxygen! It is clear from the study by Dufour, Shields, and Talbot (1982) that it is unsafe to assume that all metals scale with $O$; in particular, they find that carbon is significantly more depleted with respect to hydrogen in the SMC compared to oxygen than it is in the LMC (but see also the abundance analysis of LMC and SMC F supergiants by Russell and Bessell 1989). Does the Fe abundance scale with $\mathrm{O}$ or with C? Probably the latter, according to Dufour et al. The point here is merely that one has to be a little careful in deciding what "metallicity" to use.

Beyond the Magellanic Clouds, surveys have been made for WRs in NGC6822, IC1613, M31, and M33. Methods have included interference filter photography (Wray and Corso 1972; Corso 1975; Moffat and Shara 1983, 1987; Massey et al. 1987) and low-dispersion "grism" or "grens" searches (Westerlund et al. 1983; Bohannan, Conti, and Massey 1985; Lequeux, Meyssonnier, and Azzopardi 1987). Both of these methods rely on the detection of the CIII $\lambda 4650 / \mathrm{HeII} \lambda 4686$ band. These two methods have proven very successful at finding the strongest-lined, brightest WR stars.

However, as is well-discussed by Massey (1985), strong selection effects come into play if one wishes to determine the relative numbers of WC and WN stars within any of these galaxies. The typical equivalent width of HeII $\lambda 4686$ in WN stars is nearly a factor of 10 smaller than that of CIII $\lambda 4650$ in WC stars (compare, for example, Fig. 4 and Fig. 10 in Massey, Conti, and Armandroff 1987). To be reasonably complete for WN types requires detecting with confidence stars in which the equivalent widths of HeII $\lambda 4686$ are $40 \AA$ or so.

In order to accomplish this, an "optimized" interference filter system was designed using spectrophotometry of Galactic and Magellanic Cloud WR stars. These filters were used with CCD's on the KPNO and CTIO 4-m telescopes to survey NGC6822, IC1613, and two fields of M33 by Armandroff and Massey (1985), and to survey eight fields in M31 by Massey, Armandroff, and Conti (1986). Using DAOPHOT to obtain photometry of every star on the frames, the magnitude differences from frame to frame were computed. The error in each star's magnitude was accurately known, and therefore stars that were significantly brighter on one of the on-band exposures could be objectively identified. The advantages of this method are the greater sensitivity (leading to improved completeness), the ability to distinguish between WC and WN stars, and the fact that the method provides a quantitative measure of the significance level of each candidate; the disadvantages are the small field size obtained with the CCD and the fact that the method requires a great deal of work! In addition, the drive for relatively useful completeness implies that a certain number of candidates are bound to be "losers" (nonWR stars) if one is doing a proper job. These surveys have necessitated a great deal of 
follow-up spectroscopy, made practical primarily thanks to the implementation of multiobject fiber spectroscopy.

It has been our intention that once we understand the fraction of bona fide WR stars among our candidates as a function of significance level in Local Group galaxies (where follow-up spectroscopy is possible), these imaging surveys can be extended to more distant galaxies, such as those of the Sculptor and M81 groups. We are not quite there as yet, but almost, and you are invited to view the poster paper by Armandroff and Massey in which we show confirming spectra of many of the lower significance candidates. Despite improvements in the instrumentation, these observations are still hard. For instance, the spectrum shown of AM11 in M33, a WR candidate with a continuum magnitude of 20.5 and a significance level of $3.0 \sigma$, is the sum of three 1-hour exposures with the Mayall 4$\mathrm{m}$ telescope. This star is now definitely confirmed as a WR star, but to do so was nontrivial.

The spectroscopic evidence to date is summarized here. For NGC6822, 4 WN stars have been confirmed; there may be 1 or 2 more among the remaining candidates. There are not, at present, any WC stars living in the surveyed area of NGC6822. In IC1613, 1 WC star (previously found by spectroscopy of an HII region by D'Odorico and Rosa 1982) is known, and another star is either a WN or SNR. A few other WN's may be present, although Azzopardi, Lequeux, and Maeder (1988) say not. These small galaxies suffer from the same $\sqrt{N}$ problems discussed earlier for the SMC. The fact that the WC/WN ratio may be $1 / 0$ (i.e., infinite) in IC1613 (a galaxy with a metallicity lower than that of the SMC) seems to be conveniently ignored in the same papers that make a big deal of the small $\mathrm{WC} / \mathrm{WN}$ ratio in the SMC....

In M33 photographic surveys have found $\approx 100$ WR stars (e.g., Wray and Corso 1972; Massey et al. 1987). Based upon the data then available, Massey and Conti (1983) argued that there is a good correlation of WC to WN ratio with galactocentric distance within the plane of M33.

How good is our knowledge of the WR content of M33? Armandroff and Massey (1985) surveyed two "test" fields in M33 (from Tololo!) in order to test how well these optimized filters performed. The fields were selected only for having lots of previously known WR's. In these two test fields there were 16 previously known WR stars, of which 11 were of WC type and 5 were of WN type; e.g., a WC/WN ratio of 2.2 , twice that of the solar neighborhood. Our CCD survey found all of these plus 11 more candidates. Of these, 5 have now been confirmed spectroscopically. All the new ones are of WN type. Thus, suddenly the ratio of WC to WN in these two fields is found to be 1.1 , almost identical to that of the solar neighborhood. We may safely conclude that the $\mathrm{WC} / \mathrm{WN}$ ratio determined in such studies is affected by detection limits and small number statistics.

This of course raises an interesting point: is there in fact a gradient in the relative number of WC/WN stars in M33? The data from the CCD fields suggest that the photographic survey was at least $30 \%$ incomplete in these two fields, and of this selectively incomplete for WN stars. This is of no particular surprise, and in fact Massey and Conti (1983) state: "In selecting stars for spectroscopy, we naturally concentrated on stars with the largest differences in our blink comparison, since these were the most certain to be Wolf-Rayet stars. Thus although the overall ratio of WC to WN stars suggested by Table 1 is $1: 1$, this is likely biased in favor of the WC stars. When making the blink survey we assigned subjective values to how strongly each star blinked; using the distribution of WC and 
WN types within these categories, and allowing for the larger incidence of stars found not to be Wolf-Rayet stars in the weaker candidates, we estimate that this ratio could be as low as 1:2. Despite the factor of 2 uncertainty, we believe that the overall WC to WN ratio in M33 more closely resembles that of the solar neighborhood and the LMC than the SMC...." They go on to argue that the change in the relative numbers of WC and WN stars with galactocentric distance probably is real. However, one worry in this is that the detection of WR stars is harder near the center of M33 (where the background is higher) and thus disproportionately favors the detection of WC stars in this region. A CCD survey of the center of M33 could answer this, and in fact Drissen, Moffat, and Shara present the results of such a survey as a poster at this conference. They find a number of new WR candidates likely to be WN's, but in fact in much the same ratio as the increase we found in the two fields further out. Thus it appears that the overall gradient with galactocentric distance remains. We will return to the question of what the absolute WC/WN ratio is telling us after we consider M31.

M31 is the Local Group galaxy with more WR mythology than the others combined. "Facts and fancies" include the belief that the WR population is predominately WC's, and that the number of WR stars per unit anything is at least a factor of 10 lower than it is in the Milky Way. Let us consider what is known today about the statistics from the eight fields surveyed in M31 by Massey, Armandroff and Conti (1986).

In these eight fields there are $34 \mathrm{WR}$ candidates of various significance levels. Of these, only two were detected in the photographic survey by Moffat and Shara (1983); another five were rediscovered in their second survey (Moffat and Shara 1987). Of these 34, 19 have now been spectroscopically confirmed. Nine of these are of WC type and 10 of these are of WN type. Thus for the fields in which we feel we have reasonable completeness, the overall $\mathrm{WC} / \mathrm{WN}$ ratio is 0.9 . This must be considered an upper limit since the remaining 15 candidates are expected to be WN's on the basis of our narrow-band photometry. This ratio is already lower than that of the two M33 fields, although the oxygen abundance (relative to hydrogen) is 0.6 dex higher in M31 than in M33. So much for the WC/WN ratio being a monotonic function of metallicity.

What about the SFR/IMF massive star deficiency in M31? We find 21 spectroscopically confirmed WR's for the two AM fields in M33. The total area of these two fields is $4.4 \mathrm{kpc}^{2}$, leading to a surface density of $5 \mathrm{WR} / \mathrm{kpc}^{2}$. (The inclination of M33 has been corrected for.) Similarly, in any two "good" fields in M31 there are 8 or 9 spectroscopically confirmed WR stars. The total area (corrected for M31's inclination) is $6 \mathrm{kpc}^{2}$, leading to a surface density of $1.5 \mathrm{WR} / \mathrm{kpc}^{2}$. Thus regions rich in WR stars in M31 are down by a factor of 3, not 10, compared to rich regions in M33. For comparison, the number density near the sun is $2 \mathrm{WR}$ stars $/ \mathrm{kpc}^{2}$. Of course, the solar neighborhood is hardly an $\mathrm{OB}$ association. For comparison, the numbers (uncorrected for inclination or depth effects) are $2 \mathrm{WR} / \mathrm{kpc}^{2}$ in the $\mathrm{LMC}$, and $1 \mathrm{WR} / \mathrm{kpc}^{2}$ in the SMC.

Another part of the M31 massive star myth is that all the star formation takes place in the prominent CO ring. Van den Bergh (1964) shows the de-projected distribution of $\mathrm{OB}$ associations in M31 in his Fig. 5; clearly there are young associations that do not fall within this ring. Further surveys for WR stars are needed before we can conclude what the galaxy-wide WR content is like; high extinction will always make this a lower limit in any event. 
Ultimately we would like to know the relative numbers of WR and $O$ stars in these galaxies. This can be determined using existing technology for the Magellanic Clouds, but as recently demonstrated by Massey, Parker, and Garmany (1989), our knowledge of the massive star content of the Clouds is very incomplete. In their study of one association, NGC346, they identified 11 stars of type 06.5 or earlier; this is as many early-type $O$ stars as had previously been known in the rest of the SMC. Given that NGC6822, IC1613, M33, and M31 are 5 to 6 magnitudes further away, we face the fact that the progenitor $\mathrm{O}$ stars are a factor of 100 or more fainter. Spectroscopy of the brightest supergiants can be accomplished with existing 4-m telescopes (see, for example, the recent paper by Humphreys, Massey, and Freedman 1990), but such a sample is dominated by lowermass (10-20 $\left.\mathcal{M}_{\odot}\right)$ somewhat evolved stars. To make even a dent in this issue requires a significant amount of dedicated time on a 4-m class telescope with multi-object capability, and really an $8-\mathrm{m}$ telescope. The difficulty is that the luminosity functions are dominated by lower-mass evolved supergiants, and that spectroscopy is needed as colors and absolute visual magnitudes cannot distinguish between $20 \mathcal{M}_{\odot}$ and $100 \mathcal{M}_{\odot}$ main-sequence stars. These issues are discussed at some length in Massey (1985) and Massey (1990). To illustrate the point here we show in Fig. 1 three luminosity functions. The curve in the

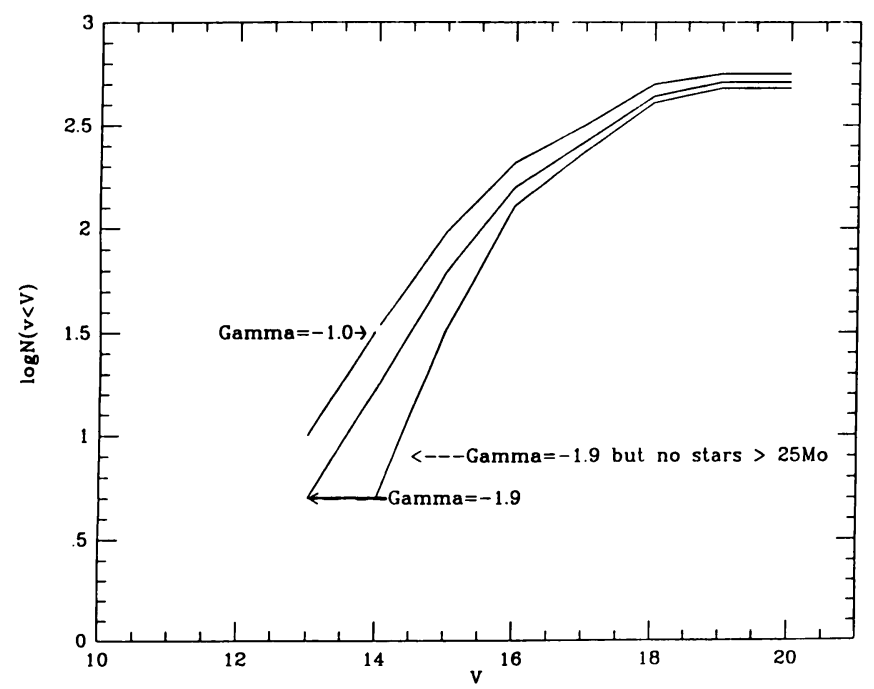

Figure 1: Luminosity functions that result from three different initial mass functions.

middle is the straight observational data taken from the Massey, Parker, and Garmany (1989) study of NGC346 in the SMC. The initial mass function was found to have a slope of $\Gamma=-1.9$. The lower slope is what results from the same data but deleting those stars whose implied masses are greater than $25 \mathcal{M}_{\odot}$. The upper curve is the luminosity function that results from the same set of stars in NGC346 but with an altered mass function $\Gamma=-1.0$, which would imply a factor of 3 more massive-star progenitors. The slopes of these luminosity functions are virtually indistinguishable. Furthermore, these represent the most optimistic case imaginable: that of an OB association in which all the stars are born at the same time. For a field population (such as what would be found in a galaxywide sample) the cooler but visually brighter $B$ supergiants would play a dominant role in the luminosity function, masking any variation at the upper mass end. Therefore, the relative numbers of WR and $O$ stars are significantly more poorly known than the 
WC/WN ratio or the surface density of WR's. We do not feel that studies of the WR/O ratio as a function of metallicity will be meaningful until the $\mathrm{O}$ star numbers result from spectroscopic surveys.

\section{THE SPECTRAL PROPERTIES OF EXTRA-GALACTIC WR STARS}

Here we are again guided by "educated intuitional whims" in what we are looking for, since we have no theory driving us in a particular direction. First let us consider the issue of late WC stars. Are there any WC stars of type WC8 or WC9 anywhere except in the Milky Way?

- None are present in the Magellanic Clouds.

- None are known in M33.

- None are known for sure in M31.

It is necessary to expound on the last point a little. Moffat and Shara $(1983,1987)$ classify several of their M31 WC stars as being WC8 or later. However, it is not clear from their spectra that CIII $\lambda 5696$ is actually stronger than CIV $\lambda 5812$ as it is in Galactic WC stars of this type, and quantitative line ratios are lacking for their data. For the stars in their sample that we have been able to observe, we find no WC stars later than WC7. It is worth noting, however, that the WC8's and WC9's in the Galaxy clearly have the weakest lines of any of the WC's and that there may therefore be selection problems (Massey, Conti, and Armandroff 1987).

Let us now consider the issue of the distribution of WC stars within M31 and M33 a little more carefully. First we will use a somewhat looser definition of what makes a late WC star "late". Massey, Conti, and Armandroff (1987) classified their WC stars using the following criteria:

WC: Neither CIII $\lambda 5696$ nor OV $\lambda 5592$ could be seen; in all cases CIV $\lambda 5812$ was visible, so these stars could not be as late as WC8 or WC9.

WCL: CIII $\lambda 5696$ could be seen and was stronger than OV $\lambda 5592$.

WCE: OV $\lambda 5592$ could be seen and was stronger than CIII $\lambda 5696$.

Thus the WCL designation would apply to any WC star of type WC6 or later. Using these simple criteria, do we see a central concentration of the WCL's in M31 or M33? Let us divide each galaxy into an "inner" and "outer" region using de-projected galactocentric distance (e.g., the distance within the galactic disk). We find the following.

TABLE 1

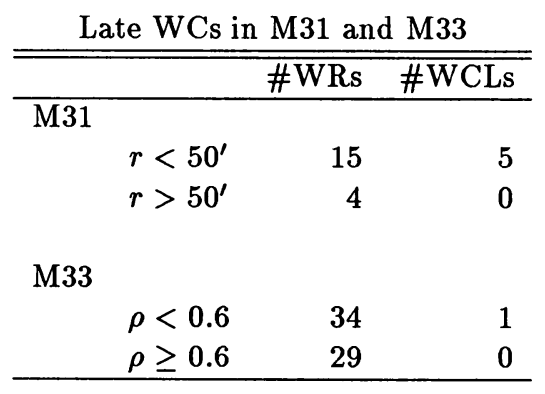

Within the inner regions of M31 we see 15 WR stars of which 5 are WCL's. In the 
outer regions there are only $4 \mathrm{WR}$ 's, and 0 is consistent with the expected number of WCL's given the small number statistics. Similarly the numbers for M33 are completely inconclusive. Thus at present we conclude there is no evidence one way or another for this particular "fact and fancy", but it is one that could be answered by simply surveying more regions in M31.

Next let us consider the more general (and interesting) question: are the stellar wind laws in extra-galactic WR stars similar to those of WR stars in our Galaxy? Since stellar winds are driven through resonance lines of highly ionized metals, we might expect that the answer is "no". On the other hand, the compositions of WR stars are mainly "home brewed" and it may be that any differences caused by different "initial metallicity" have long since been lost by the time of the WR phase. To address this question, Peter Conti developed the "inverted hockey stick" plots found in Massey, Conti, and Armandroff (1987). In these plots, the full width at half maximum (fwhm) is plotted against the log of the equivalent width. The former is something like the expansion velocity at the depth where the line is formed, and the latter is something like the wind density. Displayed in such a plot, data from Galactic and LMC stars clearly do not scatter, but fall in a relatively well-defined "inverted hockey stick". Note that the presence of an O-type companion will reduce the log ew but that the fwhm will stay roughly constant; e.g., the presence of a companion will mainly lower a point in this diagram.

Plotting the extra-galactic WR stars in this way using the new data at our disposal reveals the following:

- The Magellanic Cloud WR stars (both WC's and WN's) match the Galactic stars.

- The NGC6822 WN stars pretty well match the Galactic and Magellanic Cloud stars.

- The M33 stars (both WC's and WN's) match the Galactic and Magellanic Cloud stars.

- The M31 stars (particularly the WC's) don't match the Galactic and MC stars very well, but the data are still scant.

To this discussion can be added the fact that Hutchings, Massey, and Bianchi (1987) managed to obtain a UV spectrum of the M31 WN star OB69-WR2 with the International Ultraviolet Explorer satellite. The spectrum shows a curious absence of the strong emission lines that characterize stars of similar type in the Galaxy and Magellanic Clouds. Not even HeII $\lambda 1640$ is visible. The optical spectrum of this star shows lines which are somewhat narrow. With the more sensitive UV spectrometer on Hubble Space Telescope, it should be possible to extend such studies to many more WR stars in Local Group galaxies.

Although Massey, Conti, and Armandroff (1987) concluded that the M33 WR stars are normal and the M31 stars may be odd, recent spectroscopy by Schild, Smith, and Willis (1990) yields the opposite conclusion-that while the M31 stars in their sample appear "normal", the M33 stars are peculiar. Their reasons for concluding that the M33 stars are odd are two-fold: (1) the FWHM of the M33 WC stars in their sample appears correlated with galactocentric distance, and (2) they find that the FWHM varies within a WC subtype as a function of galactocentric distance.

We have looked for these effects in our considerably larger sample, and our independent data confirms the first of these. Figure 2 shows the distribution of WC stars in the "hockey stick" diagrams for the inner and outer regions of M33. Clearly the WC stars in the inner regions have narrower (and weaker) lines than those in the outer regions. 

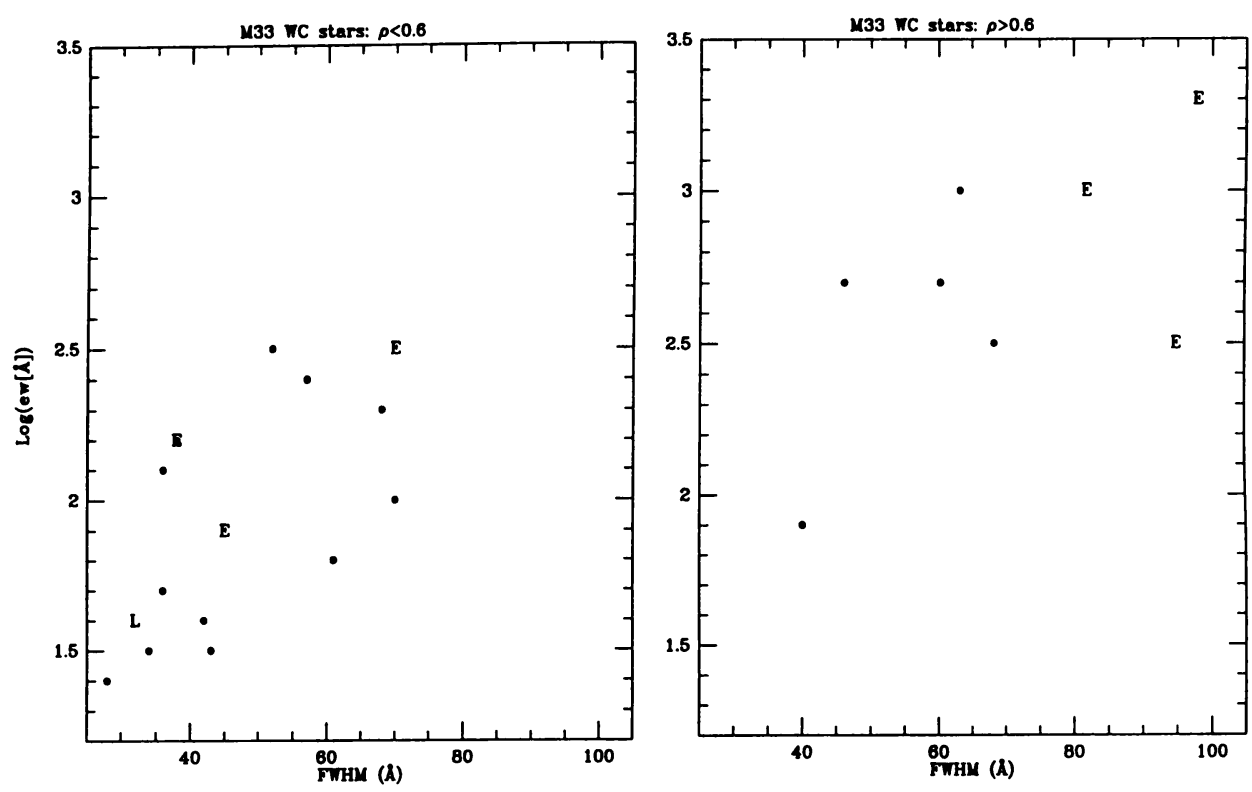

Figure 2: "Hockey stick" diagrams for M33 WC stars divided into two galactocentric distance bins; E denotes WCE's and L denotes WCL's.
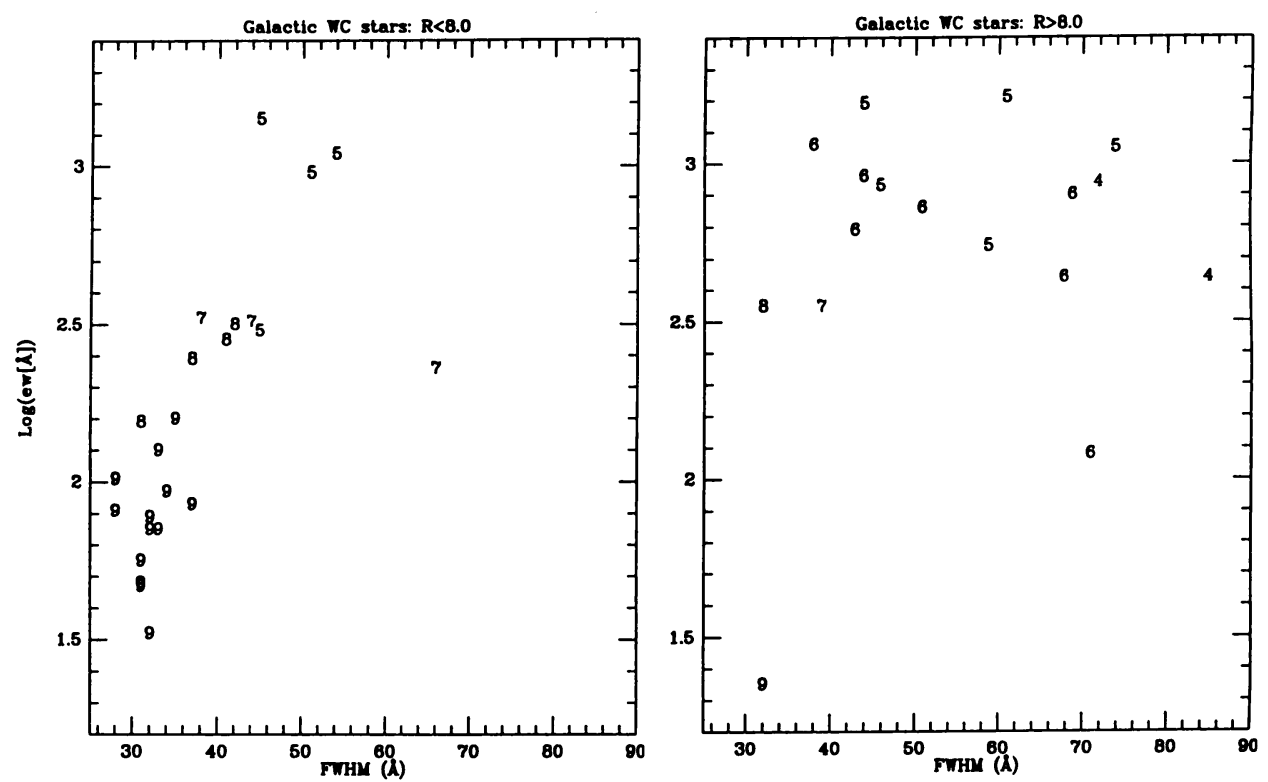

Figure 3: "Hockey stick" diagrams for Galactic WC stars with galactocentric distances less than and and greater than $8 \mathrm{kpc}$; the numerals denote each star's subtype. 
However, examining the data for the Galactic WC stars reveals an identical effect! We have taken values for the equivalent widths and fwhm's from Conti and Massey (1989); the galactocentric distances come from Conti and Vacca (1990). Figure 3 shows the data for the Galaxy broken into "inner" and "outer" regions. Clearly the situation is no different in M33 than in the Milky Way.

Massey, Conti, and Armandroff (1987) did not feel brave enough to assign numerical subtypes to the WC stars in their extra-galactic sample, so their data cannot be used to confirm or refute the second conclusion of the Schild, Smith, and Willis (1990) study that the fwhm within a subtype is dependent upon galactocentric distance within M33. However, Fig. 3 demonstrates that this is certainly the case for the Galactic data: the WC5 stars, for instance, have skinnier fwhm in the inner part of the Galaxy than they do in the outer. So if the M33 stars are similar to those in our Galaxy, we would perhaps not be surprised to see such an effect. Why are these effects present? What is this telling us about stellar wind laws and their dependence upon (say) metallicity? We do not know the answer to this for certain, but whatever it is, it appears to be at work both in M33 and in the Milky Way.

\section{REFERENCES}

Armandroff, T. E., and Massey, P. 1985, Ap. J., 291, 685.

Azzopardi, M., and Breysacher, J. 1979, Astr. Ap., 75, 120.

Azzopardi, M., Lequeux, J., and Maeder, A. 1988, Astr. Ap., 189, 34.

Barden, S. C., and Massey, P. 1988, in S. C. Barden (ed.), Fiber Optics in Astronomy, (Provo: Astron. Soc. Pacific), p. 140.

Bohannan, B., Conti, P. S., and Massey, P. 1985, Astron. J., 90, 600.

Breysacher, J. 1981, Astr. Ap. Suppl., 43, 203.

Conti, P. S. 1982, in C. W. H. de Loore and A. J. Willis (eds.), Wolf-Rayet Stars: Observations, Physics, Evolution, IAU Symp. No. 99 (Dordrecht: Reidel), p. 3.

Conti, P. S. 1988, in V. M. Blanco and M. M. Phillips (eds.) Progress and Opportunities in Southern Hemisphere Optical Astronomy, (Provo: Astron. Soc. Pacific), p. 100.

Conti, P. S., and Massey, P. 1989, Ap. J., 337, 251.

Conti, P. S., and Vacca, W. D. 1990, Astron. J., 100, 431.

Corso, G. J. 1975, Bull. A.A.S., 7, 411.

D'Odorico, S., and Rosa, M. 1982, Astr. Ap., 105, 410.

Dufour, R. J., Shields, G. A., and Talbot, Jr., R. J. 1982, Ap. J., $252,461$.

Humphreys, R. M., Massey, P., and Freedman, W. L. 1990, Astron. J., 99, 84.

Hutchings, J. B., Massey, P., and Bianchi, L. 1987, Ap. J., 322, L79.

Lequeux, J., Meyssonnier, N., and Azzopardi, M. 1987, Astr. Ap. Suppl., 67, 169.

Massey, P. 1985, Pub. A.S.P., 97, 5.

Massey, P. 1986, in C. W. H. de Loore, A. J. Willis, and P. Laskarides (eds.) Luminous Stars and Associations in Galaxies, IA U Symp. No. 116, (Dordrecht: Reidel), p. 215.

Massey, P. 1990, in C. D. Garmany (ed.) Properties of Hot Luminous Stars (Provo: Astron. Soc. Pacific), p. 30.

Massey, P., Armandroff, T. E., and Conti, P. S. 1986, Astron. J., 92, 1303.

Massey, P., and Conti, P. S. 1983, Ap. J., 273, 576.

Massey, P., Conti, P. S., Armandroff, T. E. 1987, Astron. J., 94, 1538.

Massey, P., Conti, P. S., Moffat, A. F. J., and Shara, M. M. 1987, Pub. A.S.P., 99, 816.

Massey, P., Paker, J. W., and Garmany, C. D. 1989, Astron. J., 98, 1305. 
Moffat, A. F. J., and Shara, M. M. 1983, Ap. J., 273, 544.

Moffat, A. F. J., and Shara, M. M. 1987, Ap. J., 320, 266.

Russell, S. C., and Bessell, M. S. 1989, Ap. J. Suppl., 70, 865.

Schild, H., Smith, L. J., and Willis, A. J. 1990, Astr. Ap., in press.

van den Bergh, S. 1964, Ap. J. Suppl., 9, 65.

van der Hucht, K. A., Conti, P. S., Lundstrom, I., and Stenholm, B. 1981, Space Sci. Rev., 28, 227.

Westerlund, B. E., Azzopardi, M., Breysacher, J., and Lequeux, J. 1983, Astr. Ap., 123, 159.

Wray, J. D., and Corso, G. J. 1972, Ap. J., 172, 577.

\section{DISCUSSION}

Sreenivasan: You said that WR stars in M33 and M31 are different from those anywhere else. Does this refer to the surface number densities or does it cover other aspects? (The densities you quoted later on appeared similar!).

Massey: Actually, I did not say that. I tried to diffuse those claims. Pundits in this room have claimed that the spectra M33 and M31 WR stars are unlike those in the Galaxy, but quantitative measurements have yet to demonstrate this.

Moffat: (1) In going from regions of extreme star formation (e.g., GHR's) to the field, evidence shows that the WC/WN ratio changes dramatically (e.g., the ratio in 30 Dor is $\sim 2$ to $\sim 0.1, c f$. the rest of the LMC: $\sim 20 / 80 \approx 0.25$ ). (2) While colours of $O$ stars may be degenerate when looking in galaxies where crowding and differential reddening prevail, this is not so for nearby young clusters with accurately observed colours, e.g. $\sigma(U-B) \approx 0.01$, since $(U-B)_{o}=-1.2$ for $03 \mathrm{~V}$ to -1.1 for $09 \mathrm{~V}$.

Massey: (1) Lindsey Smith maintains in her talk that WC4's dominate the integrated WR spectrum of NGC 604, the biggest $H I I$ region in the Local Group outside 30 Dor. (2) Much of the galactic calibration of $(U-B)_{0}$ with spectral type comes from heavily reddened galactic $\mathrm{OB}$ associations - as you know, there are no lightly reddened $\mathrm{O} 3 \mathrm{~V}$ stars around to provide an empirical calibration. Models also give only a tiny difference in $(U-B)_{o}$ as a function of $T_{\text {eff }}$ from $30000 \mathrm{~K}$ to $50000 \mathrm{~K}$. For a recent discussion of this, see Massey, Parker and Garmany (1989), and Massey, Garmany, Silkey and Degioza-Eastwood (1989, Astron.J. 97, 107).

de Groot: If you were born in Northern-Ireland, you would know that there is a different kind of hockey stick according to whether you are born as a boy or as a girl. It would be a hurly or a commogy stick, exactly the shape that you drew.

Massey: Actually, it was Peter Conti that came up with the phrase "inverted hockey sticks". Maybe we need something to distinguish the late WN's from the early WN's. Perhaps we can do it on the basis of sex, rather than the $N V / N I I I$ ratios. 